\title{
All-sky catalog of local radio galaxies
}

\author{
S. van Velzen ${ }^{1}$ and H. Falcke Fal, $^{1,2}$ \\ ${ }^{1}$ IMAPP, Radboud University, P.O. Box 9010, 6500 GL Nijmegen, The Netherlands \\ ${ }^{2}$ ASTRON, Dwingeloo, The Netherlands \\ ${ }^{3}$ Max-Planck-Institut für Radioastronomie Bonn, Germany
}

\begin{abstract}
The final episode in the history of black hole accretion and galaxy formation takes place in our cosmic backyard, the local universe. Within this volume must also reside the until now unknown - sources of observed ultra-high energy cosmic rays (UHECRs). A thorough study of the local universe requires full-sky coverage to obtain a sizable sample and map the matter anisotropy. We recently constructed the first catalog of radio-emitting galaxies that meets this requirement. The sample contains all radio galaxies similar to Centaurus A out to $\sim 100$ Mpc. Only $3 \%$ of the hosts of the powerful radio jets are classified as Spiral galaxies, while for non-radio galaxies of similar mass, this fraction is $34 \%$. The energy injected by radio jets per unit volume indicates that Cen A-like radio galaxies have in principle sufficient power to accelerate cosmic rays to ultra-high energies. A significantly enhanced clustering of radio-loud galaxies compared to normal galaxies of the same luminosity is observed. This indicates a causal relation between galaxy environment and jet power, independent of black hole mass.
\end{abstract}

Keywords. catalogs, galaxies: jets, galaxies: evolution, accretion, acceleration of particles

Here we highlight two applications of our catalog (van Velzen et al. 2012, available at http://ragolu.science.ru.nl), using a volume-limited sample of radio galaxies. ( $i$ ) To find potential UHE proton accelerators, we compare the equipartition $B$-field and radius $(R)$ of the radio lobes to the minimum value required for containing cosmic rays of this energy. The jet power of these sources, estimated from the total energy in the lobes $\left(\propto B^{2} R^{3}\right)$ over their dynamical time, exceeds the energy injected into UHECRs. ( $i i)$ We observe that the projected density of galaxies around radio galaxies $\left(\rho_{\mathrm{RG}}\right)$ is significantly enhanced with respect to the mean density around non-radio galaxies of the same luminosity and Hubble type $\left(\rho_{\text {matter } *}\right)$.
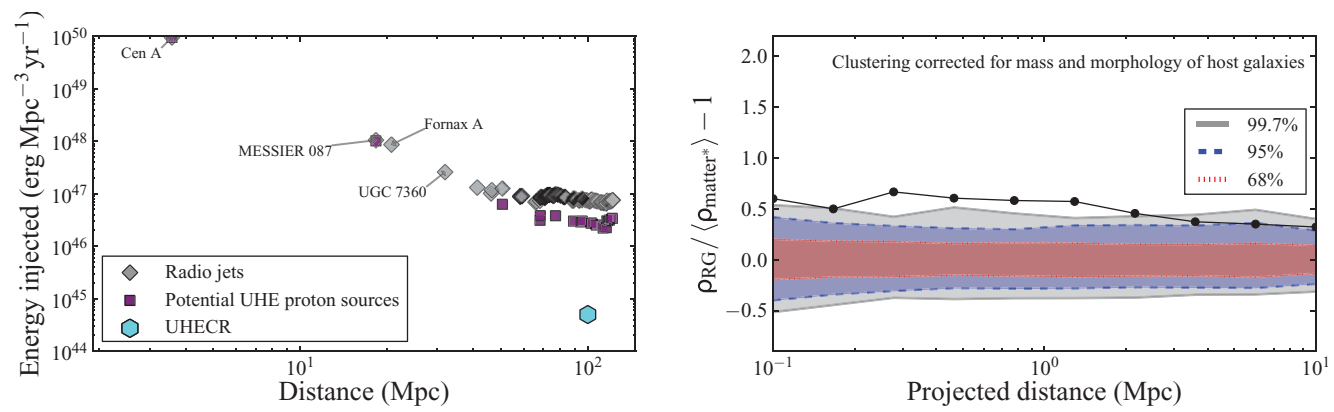

Figure 1. The energy injected in a sphere between us and the galaxy (left) and the clustering (right) of a volume-limited sample of radio galaxies $\left(L_{1 \mathrm{GHz}}>5 \times 10^{23} \mathrm{~W} \mathrm{~Hz}^{-1}, z<0.03\right)$.

\section{Reference}

van Velzen, S., Falcke, H., Schellart, P., \& Nierstenhöfer, N., Kampert K. H. 2012, A $\&$ A, 544, A18 